\title{
A model of the closed universe gravitating in 4-space
}

\author{
Zahid Zakir ${ }^{1}$
}

\begin{abstract}
A models of the closed universe as a thin 3-sphere in 4-space, gravitating along 3-sphere's radius, is formulated. Corresponding evolution equations for the dust matter and radiation in the 3 -sphere and their observational consequences are studied.

PACS: 04.20.Cv, 98.80.-k, 98.80.Jk 95.30.Sf, 97.60.Lf, 98.35.Jk, 98.54.-h, 98.80.-k, 04.60.-m

Key words: cosmological models, redshift, extra dimension

\section{Content}

Introduction

1. The evolution equation from Einstein's equations in 4-space..................................................

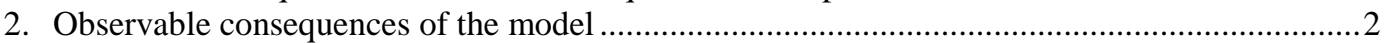

References

\section{Introduction}

In the previous papers [1] a new formulation of models of relativistic cosmology on the basis of more accurate accounting of the requirements of relativistic kinematics, especially in the closed model, was given.

This analysis has shown that former confrontations with observations of the models without dark energy were premature since did not include a number of physical requirements and effects, every one of which essentially influences to the observational predictions of models. Particularly, the progress in the closed models, which from the theoretical point of view always have been considered as most attractive ones, is that part of former arguments against them practically are disappeared.

In the present paper this analysis will be continued and it will be studied the consequences of the existence of the closed universe as 3-sphere only at embedding into 4space and, therefore, gravitating in 4-space.

The main relations of the model are presented in Sections 1-2 and in Section 3 the observational consequences are considered.

\section{The evolution equation from Einstein's equations in 4-space}

Considering the closed model of relativistic cosmology, further we will suppose that the Universe is 3 -sphere of radius $a$ embedded into the real 4-dimensional space and that its evolution is described in a rest-frame of the centre of 3 -sphere in terms of world time $t$.

The solutions of the Einstein equations $G_{i k}=\kappa T_{i k}$ for the metrics of a thin dust 3sphere in 4-space are well-known and they lead to the evolution equation:

\footnotetext{
${ }^{1}$ Centre for Theoretical Physics and Astrophyics, Tashkent, Uzbekistan; zahidzakir@ theor-phys.org
} 


$$
\frac{\dot{a}^{2}}{c^{2}}=\frac{a_{m}^{2}}{a^{2}}-1
$$

where $a_{m}$ is the radius of 3 -sphere at maximal expansion.

The speed of expansion and parameter $H_{0}$ in the model are equal to:

$$
\begin{gathered}
\dot{a} \simeq c \sqrt{\frac{a_{m}^{2}}{a^{2}}-1}, \\
H_{0}=\frac{\dot{a}_{0}}{a_{0}} \simeq c \frac{a_{m}}{a_{0}^{2}} \sqrt{1-\frac{a_{0}^{2}}{a_{m}^{2}}}=\frac{c}{a_{0} b} \sqrt{1-b^{2}},
\end{gathered}
$$

where $b=a_{0} / a_{m}$ and

$$
a_{0}=\frac{c}{H_{0}} b^{-1} \sqrt{1-b^{2}} .
$$

At taking into account the effects of relativistic kinematics [2], it is necessary to express the evolution equation (1), where the speed is a derivative on proper time, in terms of world time $t$ defined as

after which it takes the form:

$$
d \tau=d t \sqrt{1-\frac{\dot{a}^{2}}{c^{2}}}
$$

$$
\frac{\dot{a}^{2} / c^{2}}{1-\dot{a}^{2} / c^{2}}=\frac{a_{m}^{2}}{a^{2}}-1
$$

For the radiation we have, instead of (6), the evolution equation:

$$
\frac{\dot{a}^{2} / c^{2}}{1-\dot{a}^{2} / c^{2}}=\frac{a_{r m}^{3}}{a^{3}}-1
$$

where $a_{r g}^{3} \sim \rho_{r}\left(t_{0}\right) a_{0}^{4}=\rho_{r}\left(t_{m}\right) a_{m}^{4}$ with corresponding change of other relations.

\section{Observable consequences of the model}

From the equation for trajectory of a radially propagating light:

$$
d s^{2}=c^{2} d t^{2}-a^{2}(t) \cdot d \chi^{2}=0
$$

then follows:

$$
\chi_{z}=c \int_{a_{z}}^{a_{0}} \frac{d a}{a} \cdot \frac{d t}{d a}=\frac{1}{a_{m}} \int_{a_{z}}^{a_{0}} \frac{d a}{\sqrt{1-a^{2} / a_{m}^{2}}}=\arcsin (b)-\arcsin \left(b \frac{a_{z}}{a_{0}}\right),
$$

or

$$
\sin \chi_{z}=b \sqrt{1-b^{2} \frac{a_{z}^{2}}{a_{0}^{2}}}-b \frac{a_{z}}{a_{0}} \sqrt{1-b^{2}} .
$$

The redshift $z$ of wavelength $\lambda_{r}$ at reception with respect to wavelength $\lambda_{e}$ at radiation and the relation with the scale factors (in the nonrelativistic case) are defined as:

which after substitution into (10) lead to

$$
\frac{\lambda_{r}}{\lambda_{e}}=1+z, \frac{\lambda_{r}}{\lambda_{e}}=\frac{a_{0}}{a_{z}},
$$




$$
\sin \chi_{z}=\frac{b \sqrt{1-b^{2}}}{1+z}\left(\sqrt{1+\frac{2 z+z^{2}}{1-b^{2}}}-1\right) .
$$

In the case $a_{0} \ll a_{m}$, i.e. $b \ll 1$, we would obtain:

$$
\sin \chi_{z} \simeq \frac{b z}{1+z} \text {. }
$$

In the limit $z \rightarrow 0$ we have $a_{0} \sin \chi_{z} \rightarrow c / H_{0}$, which allows further to normalize the distance modulus.

Taking into account that the photometric distance is equal to $d_{p}=a_{0} \sin \chi$, for apparent luminosity $l_{F}$ and photometric distance $d_{p, 0}$ we obtain the expressions:

$$
\begin{gathered}
l_{F}=\frac{L}{4 \pi a_{0}^{2} \sin ^{2} \chi_{z}} \cdot \frac{1}{(1+z)^{2}}, \\
d_{p}=a_{0} \sin \chi_{z} \cdot(1+z)=10^{-5+(m-M) / 5} M n c,
\end{gathered}
$$

which for the distance modulus give the formula:

$$
\mu=5 \lg \left[a_{0}(1+z) \sin \chi_{z}\right]+25=5 \lg \left[b^{-1} \sqrt{1-b^{2}} \cdot(1+z) \sin \chi_{z}\right]+A,
$$

where $A=5 \lg \left(c / H_{0}\right)+25$. Substitution of Eq. (13) into (16) gives a new "distance modulus - redshift" relation for the new model:

$$
\mu=5 \lg \left[\left(1-b^{2}\right)\left(\sqrt{1+\frac{2 z+z^{2}}{1-b^{2}}}-1\right)\right]+A .
$$

In the model this formula is valid up to high enough values of $z$ as the assumptions about dust matter and insufficiency of the gravitational redshift remain be valid.

\section{References}

1. Zakir Z. (2013) Theor. Phys. Astroph. \& Cosmol. 8(2), 24, doi: 10.9751/TPAC.4518-030; 8(2), 37; doi: 10.9751/TPAC.4518-031. 